\title{
Molecular characterization of breast cancer cell response to metabolic drugs
}

\author{
Lucía Trilla-Fuertes ${ }^{1,2}$, Angelo Gámez-Pozo',2, Jorge M. Arevalillo, Mariana Díaz- \\ Almirónn, Guillermo Prado-Vázquez ${ }^{1}$, Andrea Zapater-Moros ${ }^{1}$, Hilario Navarro ${ }^{3}$, \\ Rosa Aras-López ${ }^{5}$, Irene Dapía ${ }^{6,7}$, Rocío López-Vacas ${ }^{1}$, Paolo Nanni ${ }^{8}$, Sara Llorente- \\ Armijo $^{1}$, Pedro Arias ${ }^{6,7}$, Alberto M. Borobia9 ${ }^{9}$ Paloma Maín ${ }^{10}$, Jaime Feliú ${ }^{11,12,13}$, \\ Enrique Espinosa ${ }^{11,12}$ and Juan Ángel Fresno Vara ${ }^{1,2,12}$

\footnotetext{
${ }^{1}$ Molecular Oncology and Pathology Lab, Institute of Medical and Molecular Genetics-INGEMM, La Paz University HospitalIdiPAZ, Madrid, Spain

${ }^{2}$ Biomedica Molecular Medicine SL, Madrid, Spain

${ }^{3}$ Operational Research and Numerical Analysis, National Distance Education University (UNED), Madrid, Spain

${ }^{4}$ Biostatistics Unit, La Paz University Hospital-IdiPAZ, Madrid, Spain

${ }^{5}$ Congenital Malformations Lab, Institute of Medical and Molecular Genetics-INGEMM, La Paz University Hospital, IdiPAZ, Madrid, Spain

${ }^{6}$ Pharmacogenetics Lab, Institute of Medical and Molecular Genetics-INGEMM, La Paz University Hospital-IdiPAZ, Autonomous University of Madrid, Madrid, Spain

${ }^{7}$ Biomedical Research Networking Center on Rare Diseases-CIBERER, ISCIII, Madrid, Spain

${ }^{8}$ Functional Genomics Center Zurich, University of Zurich/ETH Zurich, Zurich, Switzerland

${ }^{9}$ Clinical Pharmacology Department, La Paz University Hospital School of Medicine, IdiPAZ, Autonomous University of Madrid, Madrid, Spain Spain
} \\ ${ }^{10}$ Department of Statistics and Operations Research, Faculty of Mathematics, Complutense University of Madrid, Madrid, \\ ${ }^{11}$ Medical Oncology Service, La Paz University Hospital-IdiPAZ, Madrid, Spain \\ ${ }^{12}$ Biomedical Research Networking Center on Oncology-CIBERONC, ISCIII, Madrid, Spain \\ ${ }^{13}$ Cátedra UAM-AMGEN, Universidad Autónoma de Madrid, Madrid, Spain \\ Correspondence to: Juan Ángel Fresno Vara, email: juanangel.fresno@salud.madrid.org \\ Keywords: breast cancer; flux balance analysis; metabolism; perturbation experiments; proteomics
}

Received: October 29, $2017 \quad$ Accepted: January 03, $2018 \quad$ Published: January 08, 2018

Copyright: Trilla-Fuertes et al. This is an open-access article distributed under the terms of the Creative Commons Attribution License 3.0 (CC BY 3.0), which permits unrestricted use, distribution, and reproduction in any medium, provided the original author and source are credited.

\section{ABSTRACT}

Metabolic reprogramming is a hallmark of cancer. It has been described that breast cancer subtypes present metabolism differences and this fact enables the possibility of using metabolic inhibitors as targeted drugs in specific scenarios. In this study, breast cancer cell lines were treated with metformin and rapamycin, showing a heterogeneous response to treatment and leading to cell cycle disruption. The genetic causes and molecular effects of this differential response were characterized by means of SNP genotyping and mass spectrometry-based proteomics. Protein expression was analyzed using probabilistic graphical models, showing that treatments elicit various responses in some biological processes such as transcription. Moreover, flux balance analysis using protein expression values showed that predicted growth rates were comparable with cell viability measurements and suggesting an increase in reactive oxygen species response enzymes due to metformin treatment. In addition, a method to assess flux differences in whole pathways was proposed. Our results show that these diverse 


\section{approaches provide complementary information and allow us to suggest hypotheses about the response to drugs that target metabolism and their mechanisms of action.}

\section{INTRODUCTION}

Breast cancer is one of the most prevalent cancers in the world [1]. In clinical practice, breast cancer is divided according to three biomarkers, estrogen receptor (ER), progesterone receptor (PR) and Her2; into positive hormonal receptors $(\mathrm{ER}+)$, HER2+ and triple negative (TNBC), characterized by a lack of expression of these receptors. These biomarkers are associated with specific treatments. ER+ tumors are treated with selective ER modulator or aromatase inhibitors [2, 3] and Her2 tumors are treated with antibodies against this receptor [4]. However, TNBC tumors don't have a specific treatment. In addition to the clinical classification, molecular profiles based on mRNA expression are also established [5].

Reprogramming of cellular metabolism is a hallmark of cancer [6]. Normal cells obtain energy mainly from mitochondrial metabolism, but cancer cells show increased glucose uptake and fermentation into lactate, which is known as the Warburg effect or aerobic glycolysis [7]. Cancer cells also exhibit increased glutamine uptake to maintain the pool of nonessential amino acids and to further increase lactate production [8]. In addition, we previously observed significant differences in glucose metabolism between two of the main breast cancer subtypes: ER+ and TNBC $[9,10]$.

Metabolic alterations enable the possibility of using metabolic inhibitors as targeted drugs. Metformin (MTF), a drug for diabetes, has begun clinical trials in cancer patients [11]. It activates AMP-activated protein kinase and subsequently inhibits mammalian target of rapamycin (mTOR) [12]. On the other hand, everolimus, a rapamycin analog, has clinical activity and has been approved for use in patients with breast cancer and other tumors [13]. Rapamycin (RP) or sirolimus was the first available mammalian target of rapamycin (mTOR) inhibitor.

High-throughput mass spectrometry-based proteomics allow the quantification of thousands of proteins and the acquisition of direct information about biological process effectors. Combined with probabilistic graphical models (PGM), proteomics enables the characterization of various biological processes between different conditions using expression data without other $a$ priori information $[9,10]$.

Flux Balance Analysis (FBA) is a widely used approach for modeling biochemical and metabolic networks in a genome scale [14-16]. FBA calculates the flow of metabolites through metabolic networks, allowing the prediction of growth rates or the rate of production of a metabolite. It has traditionally been used to estimate microorganism growth rates [17]. However, with the appearance of complete reconstructions of human metabolism, FBA has been applied to other areas such as the modelling of red blood cells metabolism [18] or the study of the Warburg effect in cancer cell lines [19].

In the present study, we used proteomics and computational methods, such as PGM and a genome-scale model of metabolism analyzed using FBA, to explore the molecular consequences of metformin and rapamycin treatment in breast cancer cell lines.

\section{RESULTS}

\section{Design of the study}

We studied response against MTF and RP in six breast cancer cell lines, establishing sub-lethal doses to perform subsequent perturbation experiments. On the other hand, we studied single nucleotide polymorphisms (SNP) to check if the heterogeneity to treatment response observed among breast cancer cell lines can be associated to genetic causes. Then, perturbation experiments followed by mass spectrometry-based proteomics were done to characterize these differences at the molecular level. Differential protein expression patterns were analyzed and probabilistic graphical models (PGM) and flux balance analysis (FBA) were performed in order to characterize the molecular consequences of response against MTF and RP (Figure 1). SNP genotyping was used to study genetic variants associated with response and proteomics data were used to complement this information, study functional differences by probabilistic graphical models and improve prediction accuracy of FBA. PGM allowed characterizing differences due to the treatments at functional level and FBA was useful to study effects in the metabolic pathways. These approaches provide complementary information about genetic causes and molecular effects respectively.

\section{Breast cancer cell lines showed heterogeneous response when treated with drugs against metabolic targets}

First, we evaluated the response of ER+ and TNBC breast cancer cell lines treated with two drugs targeting metabolism, metformin (MTF) and rapamycin (RP). Cell viability was assessed for six breast cancer cell lines, three ER+ (T47D, MCF7 and CAMA1) and three TNBC (MDAMB231, MDAMB468 and HCC1143). Doseresponse curves for each drug treatment in each cell were calculated (Tables 1 and 2). A heterogeneous response was observed among breast cancer cell lines treated with a range of MTF and RP concentrations (Figure 2). Regarding RP, this heterogeneous response is related to breast cancer subtypes, showing an increased effect over $\mathrm{ER}+$ cell line viability compared with those of TNBC. 


\section{SNP genotyping of breast cancer cell lines}

SNP genotyping was performed to evaluate the association of polymorphisms to MTF and RP treatment response. Polymorphisms previously related to these drugs sensitivity were studied using a custom expression array. Regarding the response to MTF, polymorphism rs2282143 in $S L C 22 A 1$ was detected in homozygosis in MDAMB468 cells. This SNP appears with a frequency of $8 \%$ in the black population, which is the population origin of this cell line, and it is associated with decreased clearance of MTF. On the other hand, the rs628031 polymorphism, also in SLC22A1, was found in homozygosis in MCF7 and $\mathrm{HCC} 1143$ cells and in heterozygosis with a possible duplication in MDAMB468 cells. The presence of this polymorphism has been associated with a decreased response to MTF (PharmGKB; www.pharmgkb.org) (Supplementary Table 1).

Regarding the response to RP, MDAMB468 cells present a polymorphism in heterozygosis in CYP $3 A 4$ (rs2740574), which has been previously related to a requirement for an increased dose of RP as compared with a wild-type homozygote (PharmGKB; www.pharmgkb. org). Additionally, rs2868177 SNP in POR gene was detected in heterozygosis in hormone receptor-positive cell lines. The relationship of rs 2868177 with RP or another rapalog has not been previously described, although it is demonstrated that POR regulates $C Y P 3 A$ family [20]. On the other hand, rs 1045642 SNP in $A B C B 1$ gene appears in heterozygosis in all ER+ cell lines, but its effect regarding RP concentration is controversial (PharmGKB; www. pharmgkb.org) (Supplementary Table 1).

\section{Molecular characterization of breast cancer cell lines response to treatment with drugs against metabolic targets using perturbation experiments and proteomics}

SNP genotyping did not fully explain the heterogeneous response between cell lines to MTF and
RP treatment, thus we characterized the molecular basis of this heterogeneous response using proteomics in a perturbation experimental setting. Six breast cancer cell lines, treated or not with suboptimal concentrations of MTF and RP (40 mM of MTF [except for MDAMB468, in which a $20 \mathrm{mM}$ concentration was used] and $625 \mathrm{nM}$ of RP) were analyzed in duplicate using shotgun proteomics. Raw data normalization was performed adjusting by duplicate values as previously described [9]. Mass spectrometry-based proteomics allowed the detection of 4052 proteins presenting at least two unique peptides and detectable expression in at least $75 \%$ of the samples (Supplementary Table 2). No decoy protein passed through these additional filters. Label-free quantification values from these 4052 proteins were used in subsequent analyses.

We first identified proteins with differential expression between the treated and the control cells. Proteins with delta expression values between the control and treated cells higher than 1.5 or lower than -1.5 were identified for each cell line/treatment combination (Supplementary Tables 3 and 4). Then, gene ontology analyses of either increased or decreased proteins was performed. Regarding MTF treatment, MCF7 cells showed decreased expression of proteins related to mitochondria and cell cycle and increased expression of proteins involved in mitochondria and cytoskeleton as majority ontologies. T47D cells presented increased expression of proteins mostly related to mitochondria and the Golgi apparatus. CAMA1 proteins showing differential expression did not shown overrepresented functions. MDAMB231 cells showed decreased expression of proteins mostly related to mitochondria. MDAMB468 cells presented decreased expression of proteins also related to mitochondria, and increased expression in proteins mainly related to the extracellular matrix. Finally, HCC1143 showed decreased expression in proteins, mostly related to mitochondria and mRNA processing, and increased expression in proteins related to cytosol and protein binding.

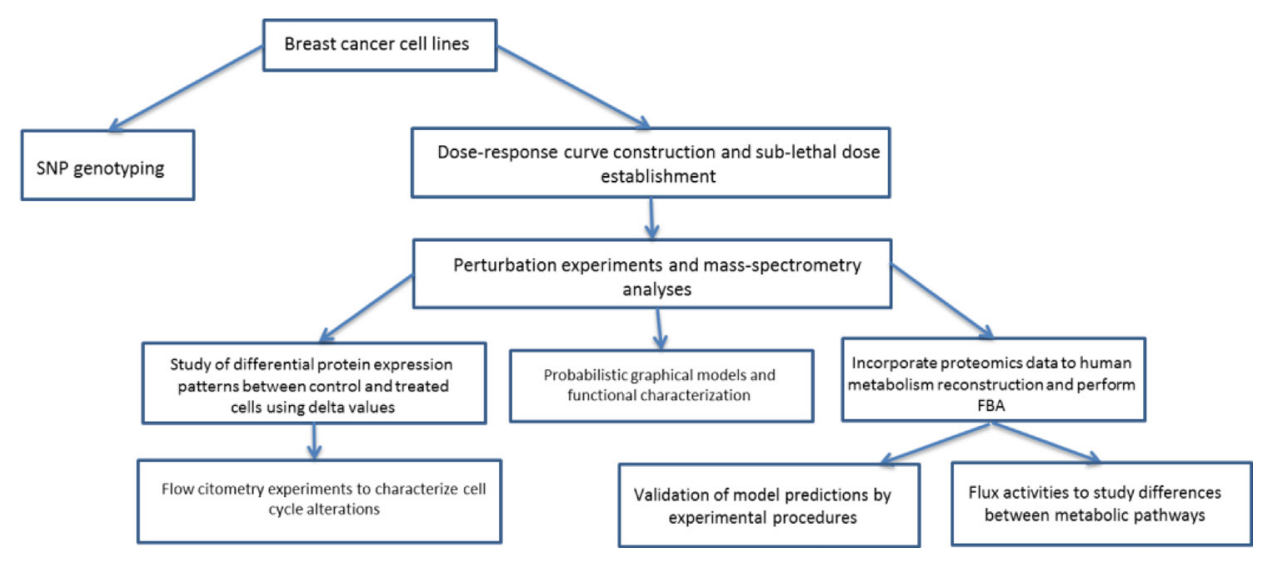

Figure 1: Workflow followed in this study. 
Table 1: Cell viability measurements in MTF treated cells

\begin{tabular}{lccccccc}
\hline MTF mM & $\mathbf{0}$ & $\mathbf{5}$ & $\mathbf{1 0}$ & $\mathbf{2 0}$ & $\mathbf{4 0}$ & $\mathbf{8 0}$ & $\mathbf{1 6 0}$ \\
\hline MCF7 & 100.00 & 135.07 & 95.00 & 61.49 & 30.61 & 28.36 & 2.47 \\
T47D & 100.00 & 85.74 & 70.15 & 59.87 & 42.11 & 7.10 & 0.00 \\
CAMA1 & 100.00 & 88.08 & 112.76 & 93.70 & 108.67 & 63.25 & 3.49 \\
MDAMB231 & 100.00 & 65.08 & 58.36 & 57.78 & 37.82 & 11.45 & 1.77 \\
MDAMB468 & 100.00 & 40.05 & 55.39 & 21.82 & 1.31 & 1.71 & 0.00 \\
HCC1143 & 100.00 & 105.48 & 85.25 & 73.19 & 52.89 & 20.49 & 0.00 \\
\hline
\end{tabular}

Cell viability measurements in six breast cancer cell lines treated with MTF $(0-160 \mathrm{mM})$. Red-white-blue color scale.

Table 2: Cell viability measurements in RP treated cells

\begin{tabular}{lcccccccc}
\hline RP nM & $\mathbf{0}$ & $\mathbf{1 5 6 . 2 5}$ & $\mathbf{3 1 2 . 5}$ & $\mathbf{6 2 5}$ & $\mathbf{1 2 5 0}$ & $\mathbf{2 5 0 0}$ & $\mathbf{5 0 0 0}$ & $\mathbf{1 0 0 0 0}$ \\
\hline MCF7 & 100.00 & 29.36 & 22.34 & 31.62 & 19.88 & 16.29 & 7.53 & 3.32 \\
T47D & 100.00 & 33.02 & 33.76 & 43.74 & 24.39 & 17.73 & 8.69 & 11.15 \\
CAMA1 & 100.00 & 70.22 & 46.25 & 45.99 & 26.28 & 22.46 & 13.45 & 7.71 \\
MDAMB231 & 100.00 & 79.92 & 82.09 & 67.84 & 62.16 & 62.43 & 31.95 & 24.50 \\
MDAMB468 & 100.00 & 48.25 & 48.51 & 71.92 & 75.75 & 52.74 & 55.31 & 4.49 \\
HCC1143 & 100.00 & 125.74 & 136.39 & 137.53 & 144.66 & 130.58 & 85.55 & 24.85 \\
\hline
\end{tabular}

Cell viability measurements in six breast cancer cell lines treated with RP $(0-10,000 \mathrm{nM})$. Red-white-blue color scale.

Differentially expressed proteins were compared with gene interaction information contained in the Comparative Toxicogenomics Database. PIR, RELA, SIRT5, CMBL, PPP4R2 and MYD88 showed decreased expression, whereas SIRT2, SERPINE1 and HTATIP2 proteins showed increased expression in cells treated with MTF in both the database and in our experiments in at least one cell line.

Concerning RP treatment, MCF7 showed decreased expression in proteins mainly related to cellular transport and an increased expression in proteins related to the mitochondrial matrix. T47D presented decreased expression in proteins involved in cell division and an increase in proteins related to lysosomes. CAMA1 had a decrease in expression of proteins associated with mRNA processing, splicing and mitochondria and an increase in the expression of proteins related to mitochondria, apoptosis processes and especially with the role of mitochondria in the apoptotic pathway. MDAMB231 had a decrease in proteins related to mRNA processing and cytoskeleton and an increase in proteins related to exosomes. MDAMB468 proteins showing differential expression did not shown overrepresented functions. Lastly, HCC1143 showed a decreased expression in proteins related to lysosomes and an increased expression in proteins related to mitochondria.

Gene interaction information contained in the Comparative Toxicogenomics Database showed a decrease in CDK4, CKS1B, COL1A1, IGFBP5, KIFC1, mTOR and $\mathrm{SCD}$ expression and an increase in CASP8, NR3C1,
PKP4, RPS27L, TEAD1 and XIAP due to RP treatment in both the database and in our experiments in at least one cell line.

Then, we applied linear regression models using protein expression data to discover molecular markers predicting the response to MTF and RP treatment. MMGT1, IDH1, PSPC1 and TACO1 showed the strongest correlation with the response to MTF (Supplementary Table 5), whereas ACADSB, CCD58, MPZL1 and SBSN correlated with the response to RP (Supplementary Table 6).

The next step was to explore molecular functions and biological pathways deregulated by MTF and RP treatment. Protein expression data from treated and untreated cells were used to build a probabilistic graphical model without other a priori information. The resulting graph was processed to seek a functional structure (Figure 3), i.e., whether the proteins included in each branch of the tree had some relationship regarding their function, as previously described [9]. Thus, we divided our graph into 36 branches and performed gene ontology analyses. Twenty-nine of them had a significant enrichment in proteins related to a specific biological function.

Functional node activity was calculated for each branch with a defined biological function using protein delta values between control and treated cells. MTF treatment caused decreased activity in mitochondria B, mRNA processing, DNA replication and ATP binding functional nodes in all cell lines (Supplementary Figure 1). In the case of RP treatment, decreased activity was 
observed in mRNA processing node activity in all cell lines (Supplementary Figure 2).

Functional node activities were then evaluated using multiple linear regression models to explore the relationship between functional deregulation and MTF/ $\mathrm{RP}$ treatment. The response to RP treatment was explained using metabolism A and B node activities (adjusted $\mathrm{R}^{2}=0.955$ ). Metabolism A node is primarily related to fatty acid biosynthesis and pyrimidine metabolism and Metabolism B node is related to glycolysis, oxidative phosphorylation and carbon metabolism (Supplementary Table 7). The response to MTF could not be predicted using this approach.

\section{Cytometry experiments showed cytostatic effects of metformin and rapamycin treatment in breast cancer cells}

The proteomics analysis workflow and gene ontology of delta values suggested that MTF and RP cause cell cycle alterations due to the recurrent replay of cell cycle category in ontology analyses. To confirm this hypothesis, flow cytometry assessment of the cell cycle was performed. MCF7 and MDAMB231 cells treated with MTF showed an increased proportion of G2/M cells when compared with the control, suggesting a cell cycle arrest in the G2 phase. However, CAMA1 cells show an increase in G1 phase percentage. Regarding RP, the ER+ cell lines MCF7 and T47D treated with RP presented an increased percentage of G0/G1 cells when compared with the control, suggesting a cell cycle arrest in G1. On the other hand, the HCC1143 cycle showed an increase in G2 percentages (Figure 4, Supplementary Table 8).

\section{Flux balance analysis predicts alterations in growth rate in metformin-treated cells}

To evaluate the impact of MTF and RP treatment on cellular metabolism, an FBA, including proteomics data from perturbation experiments, was applied to estimate cell growth rates for both control and treatment conditions. FBA can be used to evaluate a metabolic computational model to obtain a prediction of the tumor growth rate. This analysis can incorporate gene or protein expression data to improve prediction accuracy. Protein data allows constraining 2414 reactions of the 4253 reactions contained in Recon2, which have a defined gene-proteinreaction (GPR) rule, which include information of the genes/proteins involved in each enzymatic reaction. FBA predicts a lower growth rate in TNBC and MCF7 cell lines treated with MTF compared with control cells. However, it predicts a higher growth rate in the case of CAMA1 cells treated with MTF (Supplementary Table 9). FBA predicts no differences in growth rate between the control and the RP-treated cells.

\section{FBA growth predictions match with experimental data from breast cancer cell cultures}

Growth studies in ER+ (MCF7 and T47D) and TNBC (MDAMB231 and MDAMB468) cell lines were performed to validate FBA predictions using a dynamic FBA cell growth model. The starting concentration of glucose in medium $(200 \mathrm{mg} / \mathrm{dl})$ was incorporated into the dynamic FBA inputs. Initial experimental cell density was estimated by direct counting of seeded cells in the delimited area and used as a function input (MCF7= $37, \mathrm{~T} 47 \mathrm{D}=31, \mathrm{MDAMB} 231=30$ and MDAMB468 $=58$ cells respectively). Growth rate predictions were comparable with experimental measurements in cell cultures over 72 hours (Figure 5). The highest deviation in absolute values is observed in MDAMB468 cells, whereas MCF7 predictions coincided with experimental observations.

\section{Flux activity characterization}

In order to compare fluxes from complete metabolic pathways between untreated and treated cell lines, a new
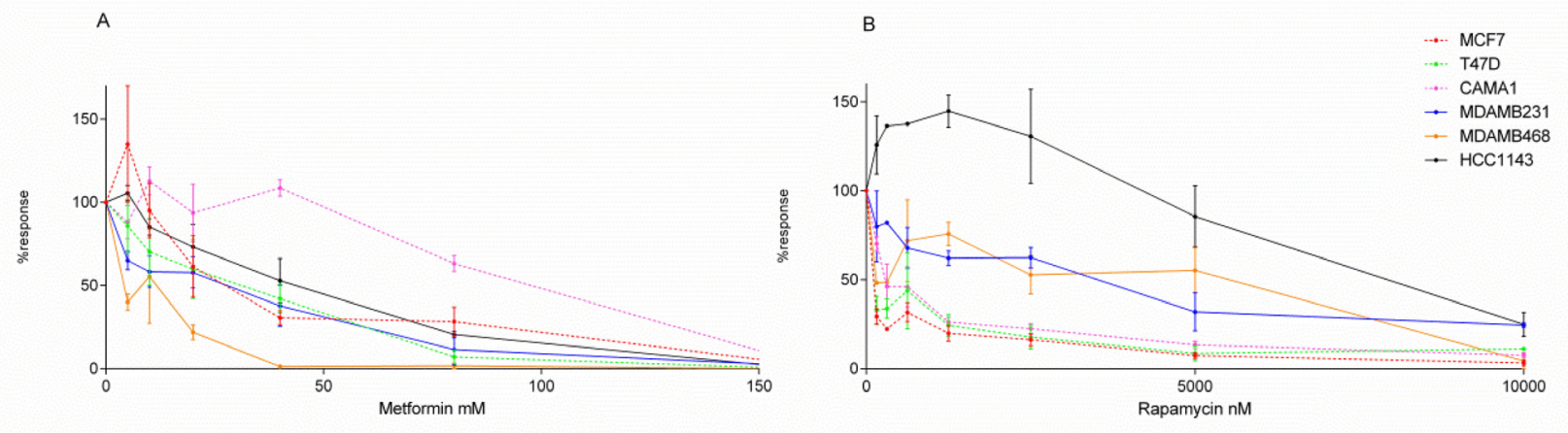

Figure 2: Dose-response curves. Dose-response curves of breast cancer cell lines treated with (A) MTF (0-160 mM) or (B) RP $(0-10,000 \mathrm{nM}) . \mathrm{ER}+$ cell lines are represented as discontinuous lines and TNBC cells as continuous lines. 
method named flux activities was proposed. Flux activities were calculated as the sum of the fluxes of each reaction in each pathway defined in the Recon2. Then, flux activities were used to build linear regression models to predict response. Pathways related to glutamate and pyruvate metabolism were related to response to MTF (adjusted $\mathrm{R}^{2}=1$ ) (Supplementary Table 10). In the case of RP, pathway fluxes that predict response against RP are cholesterol metabolism and valine, leucine and isoleucine metabolism (adjusted $\mathrm{R}^{2}=1$ ) (Supplementary Table 11).

\section{Flux analyses predict activation of ROS enzymes by metformin}

With the aim of identifying reactions that changed as a consequence of treatment, we performed a Monte Carlo analysis and chose the solution with the maximum sum of fluxes because it was representative of protein data (i.e., if a protein was measured, it indicated that the protein must be used by the cell). After that, we applied flux variability analysis (FVA) to calculate the possible maximum and minimum fluxes for each reaction, and therefore, the range of fluxes for each reaction. Next, we selected reactions showing a flux change between the control and the treated cells over $95 \%$ of this range. As long as FBA provides a unique optimal tumor growth rate, multiple combinations of fluxes can lead to this optimal value. Therefore, we confirmed that the results from the maximum flux solution were consistent throughout the multiple-solution landscape using a Monte Carlo approach to study a range of representative flux solutions from all possible solutions that optimize the tumor growth rate. Of all the candidates evaluated, we would like to highlight that FBA predicts a null catalase flux in control cells with the exception of HCC1143 cells, showing constitutive catalase activation. In MDAMB231 and MCF7 cell lines treated with MTF, the model predicts an activation of this reaction, whereas CAMA1 cells showed no response to MTF treatment regarding catalase activation (Supplementary Figure 3, Supplementary Files 1-12).

Additionally, our model predicted that superoxide dismutase (SPODM) fluxes were increased in MCF7 and HCC1143 cell lines treated with MTF, but not in MDAMB231 cells. Predictions for CAMA1 cells showed high SPODM fluxes in both control and MTF treated cells (Supplementary Figure 4 and Supplementary Files 1-12).

Finally, the Monte Carlo approach predicted an increase in nitric oxide synthase flux and, as a consequence, an increase in nitric oxide (NO) production due to MTF treatment (Supplementary Figure 5).

On the other hand, proteomics data showed an increased expression of catalase in cells treated with MTF, with the exception of the CAMA1 cell line (Supplementary Table 8). It also showed an increased

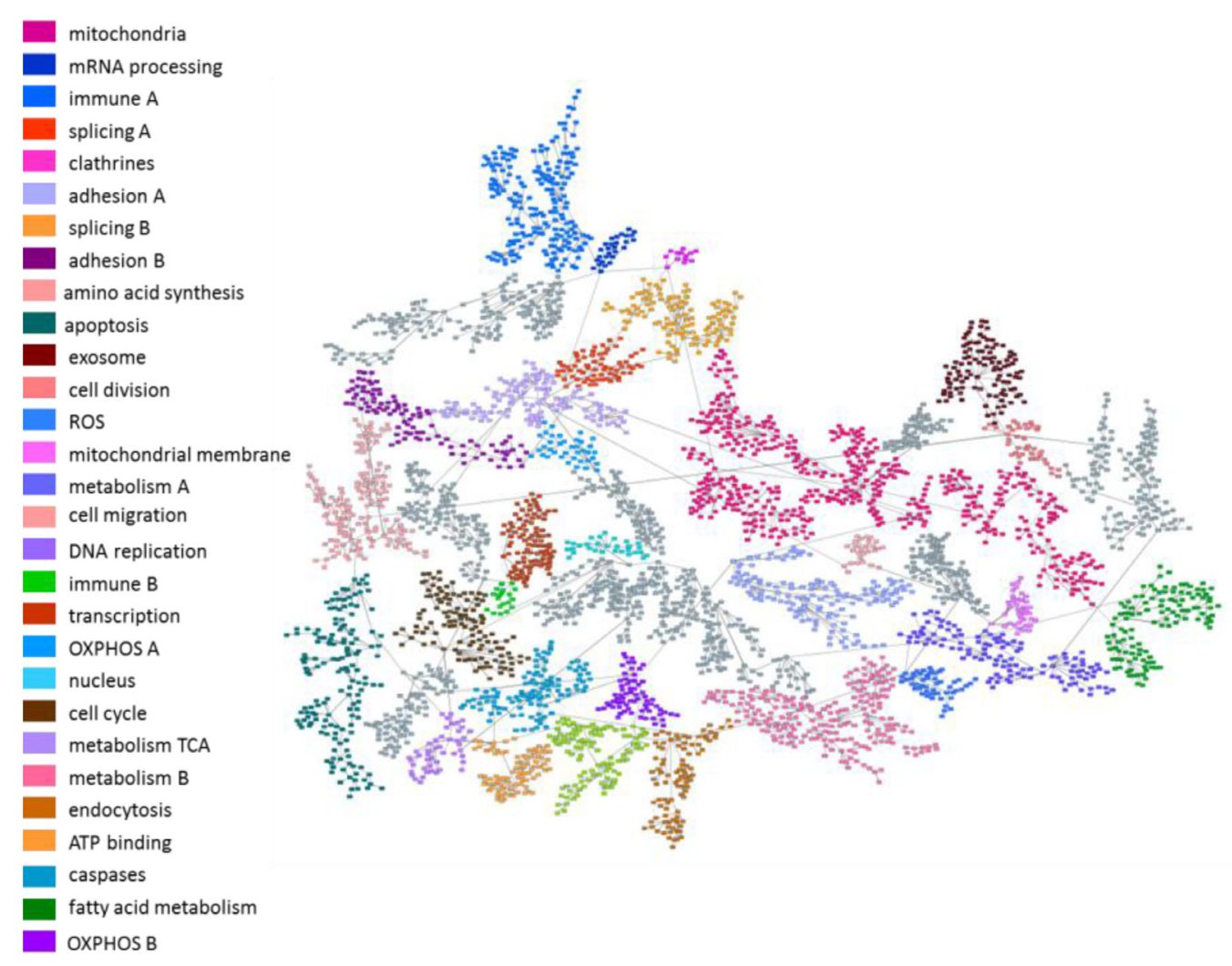

Figure 3: Probabilistic graphical model. Probabilistic graphical model using protein expression data of control and treated breast cancer cell lines. Gray nodes lack a specific function. 
expression of SPODM in cells treated with MTF, although, SPODM expression was generally lower in MDAMB231 cells than in the rest of the cell lines (Supplementary Table 12). No protein expression data from NO were obtained.
Superoxide dismutase measurements confirm superoxide dismutase activation predictions

SPODM activities were measured in the control and in the MTF-treated cells using an enzyme activity
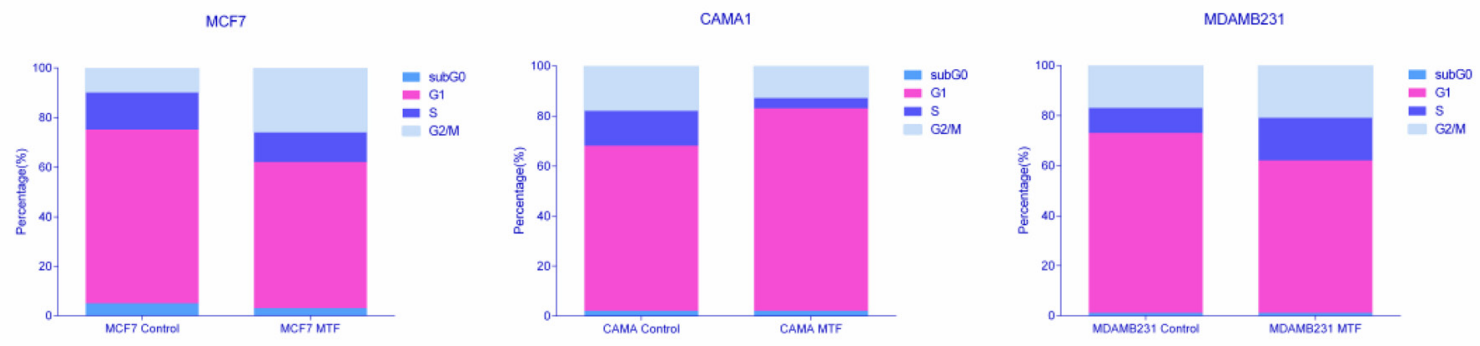

T47D
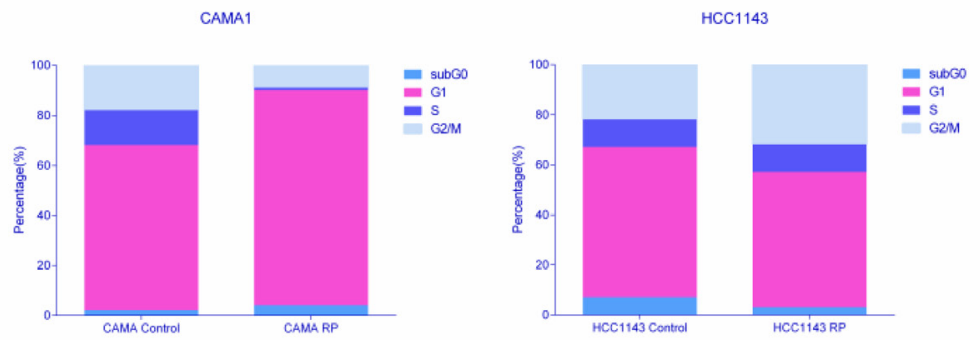

Figure 4: Percentages of cells in each cell cycle phase obtained by flow cytometry analyses.

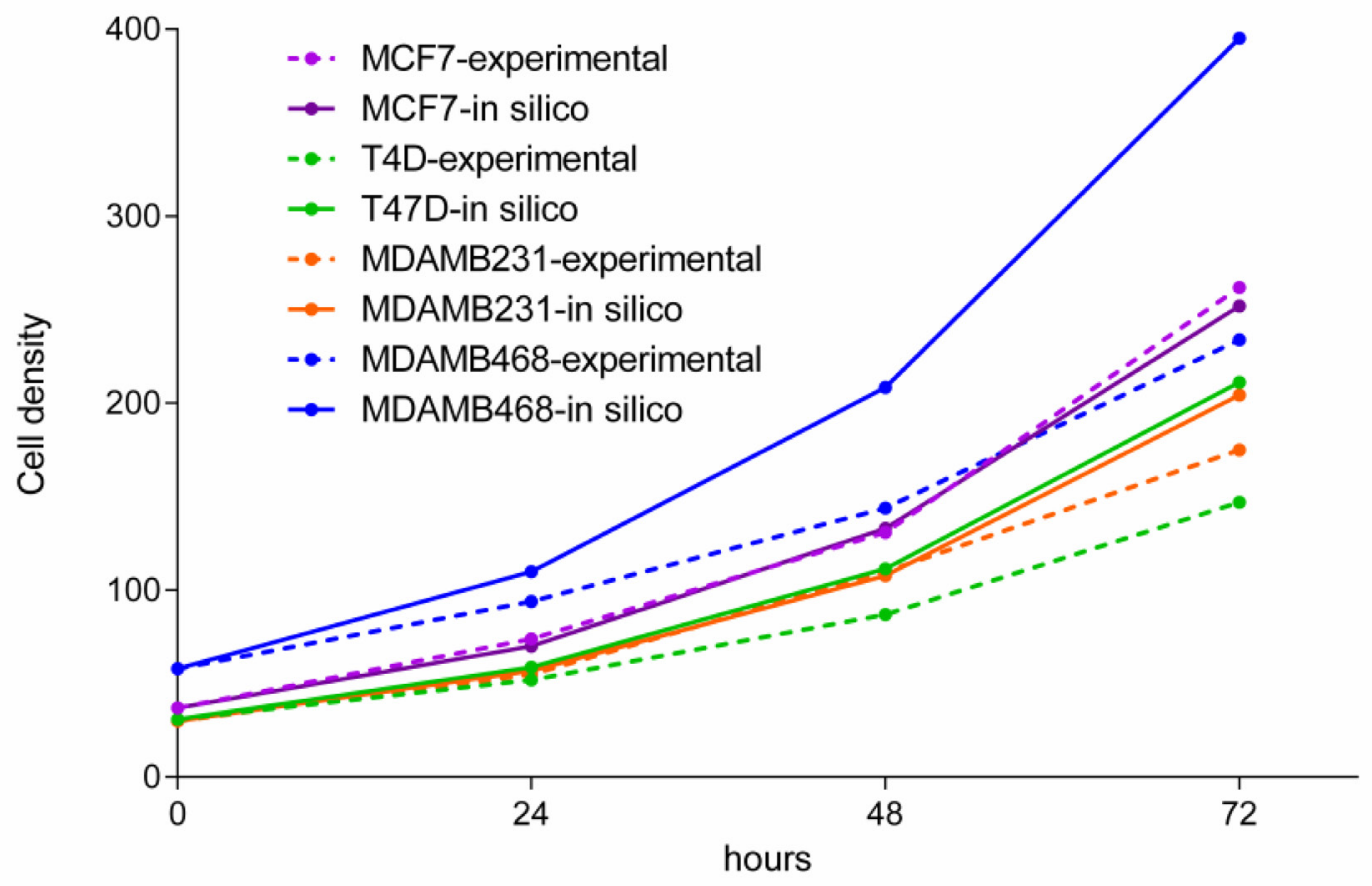

Figure 5: Experimental measurements of cell growth over 72 hours and a model simulation of growth during the same time period. 
assay. With the exception of the MCF7 cell line, model predictions were confirmed. In HCC1143, SPODM activity is similar between the control and the treated cells. On the other hand, MDAMB231 had the lowest SPODM activity, as shown in model predictions, and CAMA1 cells had the highest SPODM activity in the control and in the MTF-treated cells, as predicted in the model (Table 3).

\section{DISCUSSION}

In this study, drugs targeting metabolism elicited changes related to cell cycle and oxidative stress in breast cancer cell lines. A high-throughput proteomics approach, coupled with a metabolism computational model, was useful to predict most of these changes and propose new mechanisms of action and effects of these drugs. To our knowledge, this is the first study that combines proteomics data with this type of computational analyses to study drug's mechanism of actions in breast cancer. However, FBA was successfully used in ovarian cancer cells to propose new therapeutic targets and to study the effect of drugs targeting metabolism and their synergies [21].

In previous studies, we observed significant differences between ER+ and TNBC glucose metabolism, which showed lactate production to be higher in TNBC cells than in ER+ cells [9]. These metabolic alterations suggest the possibility of using drugs against metabolic targets in patients with breast cancer.

Our results show that breast cancer cells' response to drugs targeting metabolism is heterogeneous. MTF treatment showed a broad effect on cell proliferation, with CAMA1 cells being the most resistant to this treatment. In the case of RP, the response depends on breast cancer subtype; it is effective in ER+ cell lines but not in those of TNBCs, resembling clinical results (a derivative of RP is used in women with hormone-receptor-positive breast cancer) [13].

With the aim of studying polymorphisms that could explain this heterogeneous cell response, an SNP array was used. Therefore, the high sensitivity to MTF showed by MDAMB468 cells could be partly due to rs2282143 SNP in the $S L C 22 A 1$ carrier, which is related to decreased clearance of MTF. In addition, SLC22AI rs628031, previously associated with a poorest response against MTF, was presented as homozygotic in the MCF7 and HCC1143 cell lines. ER+ cell lines presented heterozygosis in the $A B C B 1$ rs 1045642 polymorphism, although the effects of this polymorphism in RP treatment response are not yet clear. In $C Y P 3 A 4$, rs2740574, which is related to higher requirement of sirolimus, is shown as heterozygotic in the MDAMB468 cell line.

We discovered several differences between the MTF-treated cells and the control cells. Some of these differential proteins identified matched with described interactions in the Comparative Toxicogenomics Database, such as increased expression of SIRT2 and HTATIP2 and decreased expression of SIRT5, PPP4R2 and MYD88 proteins due to MTF treatment. Increased SIRT2 protein expression induced by MTF treatment has been previously described [22]. SIRT2 also enhances gluconeogenesis, plays an important inhibitory role in inflammation and elevates ROS defense [23]. The effect of increased ROS stress response complies with our model predictions. Moreover, MTF treatment results in decreased SIRT5 expression [22]. This decrease is also related to differences observed in flux predictions between treated and control cells. It has been reported that SIRT5 is involved in the regulation of SPODM 1 activity [24], in accordance with our FBA prediction of SPODM activation in response to ROS stress in cells treated with MTF. On the other hand, TACO1, PSPC1, IDH1 and MMGT1 protein expression predict response to MTF treatment. IDH1 mutations were previously related to hypersensitivity to biguanides [25]. PGM have shown that MTF treatment caused a decreased node activity in mRNA processing, DNA replication, mitochondria B and ATP binding nodes.

We also found several differences concerning RP treatment, such as an increased expression of NR3C1 and RPS27L proteins and a decreased expression of CKS1B, COL1A1, IGFBP5, SCD, mTOR and CDK4 proteins, as previously reported [26]. CDK4/6 inhibition robustly suppressed cell cycle progression of ER+/HER2cellular models and complements the activity of limiting estrogen [27]. RP treatment also results in decreased expression of CKS1B mRNA [28]. Knockdown of CKS1 expression promotes apoptosis of breast cancer cells [29]. RP decreased expression of KIFC1 mRNA [30], whose overexpression is pro-proliferative [31]. RP treatment also results in increased activity of the NR3C1 protein [32]. NR3C1 encodes the glucocorticoid receptor, which is involved in the inflammation response and which has an anti-proliferative effect [33]. RP enhances TP73 binding to the RPS27L promoter, a direct p53 target, and consequently promotes apoptosis [34]. RP inhibits SCD mRNA expression through TP73 [35]. 17- $\beta$-estradiol induces SCD expression and the modulation of cellular lipid composition in ER+ cell lines and is necessary for estrogen-induced cell proliferation [36]. Overall, as these results showed, an anti-proliferative effect was provoked by RP treatment. Finally, RP also decreases mTOR-related protein levels [37-39]. Additionally, ACADSB, CCDC58, MPZL1 and SBSN protein expression predicts response to RP treatment. ACADSB affects valine and isoleucine metabolism [40], which is one of the pathways related to response to RP in flux activity analyses, as we will explain later. Probabilistic graphical models showed that RP treatment caused decreased node activity in mRNA processing. Additionally, metabolism A and B node activities accurately predict the response in cells treated with RP.

Proteomics coupled with gene ontology analyses allowed us to explore protein expression changes between 
Table 3: Superoxide dismutase activity assay measurements

\begin{tabular}{lc}
\hline Cell line & Superoxide dismutase activity (\%) \\
\hline MCF7 Control & $96.44 \%$ \\
MCF7 MTF & $90.76 \%$ \\
CAMA1 Control & $99.01 \%$ \\
CAMA1 MTF & $97.09 \%$ \\
MDAMB231 Control & $68.17 \%$ \\
MDAMB231 MTF & $49.82 \%$ \\
HCC1143 Control & $83.30 \%$ \\
HCC1143 MTF & $86.44 \%$ \\
\hline
\end{tabular}

The experiment was performed in triplicate and one of the representative measurements is shown.

control and treated cells, suggesting that treatment with these drugs affects cell cycle progression. Therefore, the cell cycle was further assessed using flow cytometry. A cell cycle arrest in the G2/M phase was confirmed in all the MTF-treated cells except CAMA1, in which MTF had no effect on cell viability. Additionally, ER+ cells treated with RP (but not TNBC cells) had cell cycle arrest in G0/ G1, which was confirmed at the cell proliferation level. It is known that mTOR controls cell cycle progression through S6K1 and 4E-BP1 [41]. Additionally, G0/ G1 cell cycle arrest was previously described in MCF7 cells treated with RP [42]. Therefore, MTF and RP have cytostatic effects in breast cancer cell lines and cause a cell viability reduction, coupled with a disruption of the cell cycle. However, this response is diverse between various breast cancer cell lines.

On the other hand, FBA has traditionally been used in microbiology to study microorganism growth. This approach has recently been applied to study the Warburg effect [19]. We have developed a genome-scale cancer metabolic model that uses protein expression data to predict tumor growth rate. Previous studies have described cancer metabolic models using gene expression data [19, 43, 44]. Our model, however, used a whole human metabolism reconstruction and proteomics data to improve predictive accuracy. We assessed the model reliability by growth experimental studies in ER+ (MCF7 and T47D) and TNBC (MDAMB231 and MDAMB468) cells. This approach allows new hypotheses and provides a global vision of metabolism, and has been previously used to characterize metabolism in samples from patients with breast cancer, which enables us to address clinically relevant questions [10].

Model growth rate predictions were consistent with changes detected in viability assays in the cells treated with MTF. We explored the global flux for each pathway, calculating flux activities to identify metabolic pathways showing different behavior between the MTF-treated cells and the control cells. The pathways related to response to MTF treatment were glutamate and pyruvate metabolism. The pathways related to RP treatment response were valine, leucine and isoleucine metabolism and cholesterol metabolism. Although it is difficult to make comparisons between flux patterns, pathway flux activities could be a useful approach to understanding changes between various conditions.

Moreover, by using an FVA coupled with the Monte Carlo approach, an activation of enzymes related to ROS stress response associated with MTF treatment could be predicted. Catalase and SPODM activation by MTF have been described in other scenarios $[45,46]$ and, as previously mentioned, concurs in most cases with differences shown in protein expression, although this relationship is not always direct. For instance, SPODM showed a 1.25-fold increase in protein expression, but no increment at the flux level, because fluxes are conditioned not only by their own restrictions, but also by bounds from adjacent reactions. In addition, catalase and SPODM fluxes appear to be related to cell viability. For instance, CAMA1 cells treated with MTF did not show an increased catalase flux, perhaps due to the discrete effect of MTF treatment on CAMA1 viability. Some of these predictions have been verified in the SPODM activity assay. In general, SPODM activity measurements were consistent with FBA predictions. Variations between FBA predictions and SPODM activities could be due to the fact that FBA only take into account metabolic pathways. On the other hand, our model predicts an increase in nitric oxide synthase flux in MCF7 cells treated with MTF, as has been previously described in diabetic rats [47]. An increase in nitric oxide synthase implies a higher NO concentration, related to apoptosis processes and cytostatic effects in tumor cells, whereas low NO concentrations are associated with cell survival and proliferation [48]. This nitric oxide synthase activation could be related to the reduced proliferation observed in MCF7 cells treated with MTF. The fact that this effect was only predicted in MCF7 could be due to heterogeneity in the response mechanisms against this drug in various cellular contexts, and could be related to the observed differences in cell proliferation. It is remarkable that although no information about nitric oxide synthase abundance was provided by proteomics, our model reflects differences at the flux level in this 
process, suggesting that both approaches, proteomics and flux balance analysis, offer complementary information.

To summarize our results, mitochondria and ATP binding node activities calculated by PGM functional nodes suggested that MTF effect takes place at mitochondria, a well-known fact [49]. As shown in FBA results, it also appears to increase ROS enzymes. Additionally, in MCF7 cells, an increase of nitric oxide synthase was predicted. Susceptibility to MTF treatment shown by MDAMB468 cells could be related to a SLC22A1 SNP. As consequence of these events, MTF caused a heterogeneous effect on cell proliferation, consistent with a cell cycle arrest in the G2/M phase. .

On the other hand, RP treatment exerts greater effect on the cell proliferation of ER+ cells, mediated by a G0/ G1 cell cycle arrest, as previously described [25]. This susceptibility of ER+ cell lines to RP treatment could be due to a SNP related to higher drug concentration. Finally, our results suggest that valine and isoleucine metabolism could be deregulated by RP treatment.

Our study has some limitations. FBA provides an optimal biomass value, but multiple combinations of fluxes leading to this optimum are possible, making assessing differential pathways between conditions difficult. In our study, this limitation was solved using resampling techniques; however, improvement of computational processes is still necessary. Regarding proteomics experiments, although they can improve model accuracy, because they allow direct measurement of enzyme levels, at this moment this approach can only provide values for about $57 \%$ of Recon 2 reactions with the known GPR rule. Gene expression, however, with the limitation of being an indirect measurement of enzyme abundance, provides almost the full picture. Strikingly, FBA was not able to reflect cell viability changes due to RP treatment. Despite the potential of the FBA approach, it only takes into account differences at the metabolic level. It is well known that mTOR inhibition leads to massive changes in cell homeostasis; thus, it appears reasonable that modeling changes at the metabolism level alone could not predict these differences.

In this study, we propose a workflow to study response against drugs targeting metabolism using different experimental and computational methods that allow proposing new hypotheses and characterizing this response at molecular, functional and metabolic levels providing a whole vision of the process. Moreover, we have characterized differential protein expression patterns between cells treated with drugs targeting metabolism and control cells. We have also developed a computational workflow to evaluate the impact of metabolic alterations in tumor and cell growth rates, using proteomics data. Growth rates predicted by our model matched the viability results observed in vitro with drug exposure. In addition, probabilistic graphical models are useful to study effects related to biological processes instead of considering individual protein or gene expression patterns. Our holistic approach shows that various analyses provide complementary information, which can be used to suggest hypotheses about drug mechanisms of action and response that deserve subsequent validation. Finally, this type of analysis, when fully developed and validated, could be used to study metabolic patterns from tumor samples with a different response against drugs targeting metabolism.

\section{MATERIALS AND METHODS}

\section{Cell culture and reagents}

The ER+ breast cancer cell lines MCF7, T47D and CAMA1 and the triple-negative breast cancer cell lines MDAMB231, MDAMB468 and HCC1143 were cultured in RPMI-1640 medium with phenol red (Biological Industries), supplemented with $10 \%$ heat-inactivated fetal bovine serum (Gibco), $100 \mathrm{mg} / \mathrm{mL}$ penicillin (Gibco) and $100 \mathrm{mg} / \mathrm{mL}$ streptomycin (Gibco). All the cell lines were cultured at $37^{\circ} \mathrm{C}$ in a humidified atmosphere with $5 \%(\mathrm{v} / \mathrm{v}) \mathrm{CO}_{2}$ in the air. The MCF7, T47D and MDAMB-231 cell lines were kindly provided by Dr. Nuria Vilaboa (La Paz University Hospital, previously obtained from ATCC in January 2014). The MDAMB468, CAMA1 and $\mathrm{HCC} 1143$ cell lines were obtained from ATCC (July 2014). Cell lines were routinely monitored in our laboratory and authenticated by morphology and growth characteristics, tested for Mycoplasma and frozen, and passaged for fewer than 6 months before experiments. The MTF (Sigma Aldrich D150959) and RP (Sigma Aldrich R8781) were obtained from Sigma-Aldrich (St. Louis, MO, USA).

\section{Cell viability assays}

The cells were treated with MTF and RP at a range of concentrations to establish an $\mathrm{IC}_{50}$ for each cell line. Approximately 5000 cells per well were seeded in 96well plates. After $24 \mathrm{~h}$, an appropriate concentration of drug was added to the cells, which were incubated for a total of $72 \mathrm{~h}$. Untreated cells were used as a control. The CellTiter 96 AQueous One Solution Cell Proliferation Assay (Promega) kit was used for the quantification of cell survival after exposure to the drugs. After 72 $\mathrm{h}$ of incubation with the drug, CellTiter 96 AQueous One Solution was added to each well following the manufacturer's instructions, and absorbance was measured on a microplate reader (TECAN). Experiments were performed in triplicate. $\mathrm{IC}_{50}$ values were calculated using the Chou-Talalay method [50].

\section{DNA extraction and SNP genotyping}

DNA was extracted from untreated cells using the ISOLATE II RNA/DNA/Protein Kit (BIOLINE) 
following manufacturer's instructions. We used TaqMan OpenArray technology on a QuantStudio 12K Flex Real-Time PCR System (Applied Biosystems ${ }^{\circledR}$ ) with a custom SNP array format, which allows simultaneous genotyping of 180 SNPs in major drug metabolizing enzymes and transporters (PharmArray ${ }^{\circledR}$ ). Information about the pharmacogenetic variants associated with $\mathrm{RP}$ and MTF response was gathered mostly from the variant and clinical annotations in the Pharmacogenomics Knowledge Base (PharmGKB; www.pharmgkb.org). The final selection of SNPs for our study was as follows: rs2032582, rs1045642, rs3213619 and rs1128503 in the $A B C B 1$ gene; rs55785340, rs4646438 and rs2740574 in CYP3A4; rs776746, rs55965422, rs10264272, rs41303343 and rs41279854 in CYP3A5; rs1057868 and rs2868177 in POR for RP; and rs55918055, rs36103319, rs34059508, rs628031, rs4646277, rs2282143, rs4646278, rs 12208357 in SLC22A1 and rs316019, rs8177516, rs8177517, rs8177507 and rs8177504 in SLC22A2 for MTF. Molecular analyses for rs34130495 and rs2740574 were performed by classic sequencing because these probes were not originally included in our custom SNP array design.

\section{Perturbation experiments}

Suboptimal concentrations $\left(\mathrm{IC}_{70}\right.$ or higher) were chosen in order to perform perturbation experiments (MTF 40 mM except for MDAMB468 20 m, RP $625 \mathrm{nM}$ ). Experiments were done per duplicate for each condition. Approximately 500,000 cells per well were seeded in 6-well plates. Twenty-four hours later, drugs against metabolism were added. After additional $24 \mathrm{~h}$, proteins were extracted using the ISOLATE II RNA/DNA/Protein Kit (BIOLINE). Protein concentration was determined using the MicroBCA Protein Assay Kit (Pierce-Thermo Scientific). Protein extracts $(10 \mu \mathrm{g})$ were digested with trypsin (Promega) (1:50). Peptides were desalted using in-house-produced $\mathrm{C} 18$ stage tips, then dried and resolubilized in $15 \mu \mathrm{l}$ of $3 \%$ acetonitrile and $0.1 \%$ formic acid for MS analysis.

\section{Liquid chromatography - mass spectrometry shotgun analysis}

Mass spectrometry analysis was performed on a $\mathrm{Q}$ Exactive mass spectrometer coupled to a nano EasyLC 1000 (Thermo Fisher Scientific). Solvent composition at the two channels was $0.1 \%$ formic acid for channel A; and $0.1 \%$ formic acid, $99.9 \%$ acetonitrile for channel B. For each sample, $3 \mu \mathrm{L}$ of peptides were loaded on a selfmade column $(75 \mu \mathrm{m} \times 150 \mathrm{~mm})$ packed with reversephase C18 material (ReproSil-Pur 120 C18-AQ, $1.9 \mu \mathrm{m}$, Dr. Maisch $\mathrm{GmbH}$ ) and eluted at a flow rate of $300 \mathrm{~nL} /$ min at a gradient from $2 \%$ to $35 \% \mathrm{~B}$ in $80 \mathrm{~min}, 47 \% \mathrm{~B}$ in $4 \mathrm{~min}$ and $98 \% \mathrm{~B}$ in $4 \mathrm{~min}$. Samples were acquired in a randomized order. The mass spectrometer was operated in data-dependent mode, acquiring a full-scan MS spectra $(300-1700 \mathrm{~m} / \mathrm{z})$ at a resolution of 70,000 at $200 \mathrm{~m} / \mathrm{z}$ after accumulation to a target value of 3,000,000, followed by higher-energy collisional dissociation (HCD) fragmentation on the 12 most intense signals per cycle. The HCD spectra were acquired at a resolution of 35,000 using normalized collision energy of 25 and a maximum injection time of $120 \mathrm{~ms}$. The automatic gain control was set to 50,000 ions. Charge state screening was enabled, and single and unassigned charge states were rejected. Only precursors with intensity above 8300 were selected for MS/MS (2\% underfill ratio). Precursor masses previously selected for MS/MS measurement were excluded from further selection for $30 \mathrm{~s}$, and the exclusion window was set at $10 \mathrm{ppm}$. The samples were acquired using internal lock mass calibration on m/z 371.1010 and 445.1200.

\section{Protein identification and label-free protein quantification}

The acquired raw MS data were processed by MaxQuant (version 1.4.1.2), followed by protein identification using the integrated Andromeda search engine. Each file is kept separate in the experimental design to obtain individual quantitative values. The spectra were searched against a forward Swiss-Prot human database, concatenated to a reversed decoyed FASTA database and common protein contaminants (NCBI taxonomy ID9606, release date 2014-05-06). Methionine oxidation and $\mathrm{N}$-terminal protein acetylation were set as variable modification. Enzyme specificity was set to trypsin/P allowing a minimal peptide length of 7 amino acids and a maximum of two missed cleavages. Precursor and fragment tolerance was set to $10 \mathrm{ppm}$ and $20 \mathrm{ppm}$, respectively, for the initial search. The maximum false discovery rate (FDR) was set to 0.01 for peptides and 0.05 for proteins. Label-free quantification was enabled, and a 2-minute window for match between runs was applied. The requantify option was selected. For protein abundance, the intensity (Intensity) as expressed in the protein groups file was used, corresponding to the sum of the precursor intensities of all identified peptides for the respective protein group. Only quantifiable proteins (defined as protein groups showing two or more razor peptides) were considered for subsequent analyses. Protein expression data were transformed (hyperbolic arcsine transformation), and missing values (zeros) were imputed using the missForest $\mathrm{R}$ package [51]. The protein intensities were normalized by scaling the median protein intensity in each sample to the same values. Then values were $\log _{2}$ transformed.

All the mass spectrometry raw data files acquired in this study may be downloaded from Chorus (http:// chorusproject.org) under the project name "Metabolism targeting in breast cancer cells". The peptides output file 
from the MaxQuant analysis is provided as supplementary material (Supplementary Table 2).

\section{Gene ontology analyses}

Protein expression patterns were compared between the control and treated cells, and deltas were calculated for each drug in each cell line by subtracting control protein expression from treated cell protein expression values. Gene ontology analyses were performed to determine differential functions between the control and the treated cells. For this, we selected protein showing a change in expression values (delta) higher than 1.5 or lower than -1.5 ; this delta value was calculated for each protein as the treated cell expression value minus the control cell expression value. Protein-to-gene ID conversion were performed using Uniprot (http://www.uniprot.org) and DAVID [52]. The gene ontology analyses were performed using the functional annotation chart tool provided by DAVID. We used "homo sapiens" as a background list and selected only GOTERM-FAT gene ontology categories and Biocarta and KEGG pathways. Functional categories with $p<.05$ and a FDR below 5\% were considered as significant.

\section{Probabilistic graphical models, functional node activity measurements and response predicted models}

Network construction was performed using probabilistic graphical models compatible with high dimensional data using correlation coefficients as associative measures as previously described [9]. To build this model, protein expression data without other a priori information was used. grapHD package [53] and R v3.2.5 [54] were employed to build the model.

The resulting network was split into several branches and a gene ontology analysis was used to explore the major biological function for each branch, defining functional nodes. Again, gene ontology analyses were performed in DAVID webtool [52] using "homo sapiens" as background and GOTERM-FAT, Biocarta and KEGG categories. Functional node activity was calculated as the mean delta between treated and untreated cells of all proteins related to the assigned majority node function. In order to relate drug response to functional processes, multiple linear regression models were performed using IBM SPSS Statistics.

\section{Cytometry experiments}

Some 500,000 cells were seeded in each well per duplicate. Twenty-four hours later, drugs were added and, after $72 \mathrm{~h}$, the cells were fixed in ethanol and marked with propidium iodide. Cells were acquired using a FACScan cytometer equipped with a blue laser at a wavelength of
$488 \mathrm{~nm}$. Acquired data were analyzed using BD CellQuest Pro software, first filtering cells by size and complexity in order to exclude debris, and then excluding doublets and triplets by FL2-W/FL2-A.

\section{Flux balance analysis and E-flux algorithm}

FBA was used to build a metabolic computational model that predicts growth rates. FBA calculates the flow of metabolites through metabolic networks and predicts growth rates or the rate of production of a given metabolite. It was performed using the COBRA Toolbox v2.0 [55] available for MATLAB and the human metabolism reconstruction Recon2 [56]. MATLAB $\mathrm{R} 2014 \mathrm{~b}$ and glpk solver were used. The biomass reaction proposed in Recon2 was used as an objective function representative of growth rate in tumor cells. Proteomics expression data were included in the model by solving GPR rules and using a modified E-flux algorithm [57]. Measuring GPR rule estimation values was performed using a variation of the method described by Barker et al. [58]. As described in previous works [10], the mathematical operations used to calculate the numerical value were the sum of "OR" expressions and the minimum of "AND" expressions. Finally, the GPR rule values, $a j$, were normalized to a $[0,1]$ interval, using a uniform distribution formula. The normalized values have been used to establish both new lower and upper reaction bounds. If the reaction is irreversible the new bounds are 0 and $a j$, and if the reaction is reversible the new bounds are $-a j$ and $a j$ (Supplementary Figure 6, Supplementary File 13).

\section{Metabolism model validation}

In order to validate model predictions we used dynamic FBA, which allows the prediction of cell growth during a period of time [43] and experimental growth studies of cell lines were performed. Dynamic FBA consists of an iterative approach based on a quasisteady state assumption [59]. MCF7, T47D, MDAMB468 and MDAMB231 were seeded at an initial cell density of 1,000,000 cells. Cells within the same area were counted once a day for 3 days. To perform the dynamic FBA, experimental cell density at the beginning and experimental measured glucose concentration in the medium were used as inputs in the computational simulation. Glucose presented in the medium was measured using an ABL90 FLEX blood analyzer (Radiometer). dynamicFBA function implemented in COBRA Toolbox was used. The simulation was performed for a time of 72 hours as the cell density experimental measurements. 


\section{Flux activities}

With the aim of comparing the activity of the various pathway fluxes between the control and the treated cells, flux activity was calculated for each condition. Flux activity was defined by the sum of fluxes for all reactions involved in one pathway as defined in the Recon2. Then, linear regression models were performed.

\section{Flux variability analysis and the Monte Carlo approach}

One obvious limitation to the FBA approach is that this analysis provides a unique optimal tumor growth rate, however, multiple combinations of fluxes can lead to this optimal value. In order to evaluate a representative sample of these multiple solutions, a Monte Carlo approach [60] was used to compare differential fluxes between treated and untreated cells. The solution showing the maximum sum of all the fluxes was then used to calculate the flux change between the control and the treated cells. This criterion was selected under the premise that if a protein was experimentally measured it was because that protein was going to be used by the cell; thus, maximum flux solution picks up all measured proteins. On the other hand, FVA provides the possible maximum and minimum fluxes for each reaction; therefore, the flux range for each reaction. This range was used to calculate the flux change between the control and the treated cells for a given reaction as a percentage of the flux range for that reaction. Reactions showing a flux change between the control and the treated cells over $95 \%$ of this range were identified for each condition. Monte Carlo results for these reactions were used to check if maximum solution flux is representative of the most frequent solution flux for this reaction.

\section{Superoxide dismutase activity assay}

To validate some of our model hypotheses, a SPODM activity assay was performed in triplicate, using the Superoxide Dismutase Assay Kit (Sigma-Aldrich, 19160). Some 500,000 cells per well were seeded, and after $24 \mathrm{~h}$, MTF was added at $40 \mathrm{mM}$ (except for the MDAMB468 cell line, in which a $20 \mathrm{mM}$ concentration was used). Twenty-four hours later, SPODM activities were measured following the manufacturer's instructions.

\section{Statistical analyses and software suites}

Dose-response curves were constructed with GraphPad Prism 6. Gene and protein interactions for each drug were obtained from the Comparative Toxicogenomics Database (http://ctdbase.org/) [61]. Linear and multiple regression models were built using IBM SPSS Statistics.

\section{Abbreviations}

MTF: Metformin; RP: Rapamycin; ER+: Hormonereceptor positive; TNBC: Triple negative; SNP: Single Nucleotide Polymorphism; FBA: Flux Balance Analysis; ROS: reactive oxygen species; GPR: Gen-ProteinReaction; FVA: Flux Variability Analysis; NO: nitric oxide; SPODM: superoxide dismutase.

\section{Author contributions}

All the authors have directly participated in the preparation of this manuscript and have approved the final version submitted. LT-F and RL-V performed the viability experiments and prepared the proteomics samples. LT-F performed the FBA. PN contributed the mass spectrometry data. JMA, HN and PM contributed the probabilistic graphical models. MD-A contributed the GPR rule calculation. LT-F, RL-V and RA-L contributed the enzyme activity assays. LT-F, GP-V, AZ-M and SL-A performed the statistical analysis, the probabilistic graphical model interpretation and the gene ontology analyses. ID, PA and AMB contributed the SNP study. LT-F drafted the manuscript. LT-F, AG-P, JAFV, JF and EE conceived of the study and participated in its design and interpretation. AG-P, JAFV, and EE supported the manuscript drafting. AG-P and JAFV coordinated the study. All the authors have read and approved the final manuscript.

\section{ACKNOWLEDGMENTS}

The cytometry experiments were performed at the Cytometry and Fluorescence Microscopy Center, Faculty of Chemistry, Complutense University of Madrid, Spain.

\section{CONFLICTS OF INTEREST}

JAFV, EE and AG-P are shareholders in Biomedica Molecular Medicine SL. LT-F is an employee of Biomedica Molecular Medicine SL. The other authors declare no competing interests.

\section{FUNDING}

This study was supported by Instituto de Salud Carlos III, Spanish Economy and Competitiveness Ministry, Spain and co-funded by the FEDER program, "Una forma de hacer Europa" (PI15/01310). LT-F is supported by the Spanish Economy and Competitiveness Ministry (DI-15-07614). The funders had no role in the study design, data collection and analysis, decision to publish or preparation of the manuscript. 


\section{REFERENCES}

1. Siegel RL, Miller KD, Jemal A. Cancer statistics, 2015. CA Cancer J Clin. 2015; 65:5-29.

2. Fisher B, Costantino J, Redmond C, Poisson R, Bowman D, Couture J, Dimitrov NV, Wolmark N, Wickerham DL, Fisher ER, Margolese R, Robidoux A, Shibata H, et al. A randomized clinical trial evaluating tamoxifen in the treatment of patients with node-negative breast cancer who have estrogen-receptor-positive tumors. N Engl J Med. 1989; 320:479-84. https://doi.org/10.1056/ nejm198902233200802.

3. Buzdar A, Jonat W, Howell A, Jones SE, Blomqvist C, Vogel CL, Eiermann W, Wolter JM, Azab M, Webster A, Plourde PV, and Arimidex Study Group. Anastrozole, a potent and selective aromatase inhibitor, versus megestrol acetate in postmenopausal women with advanced breast cancer: results of overview analysis of two phase III trials. J Clin Oncol. 1996; 14:2000-11.

4. Slamon DJ, Clark GM, Wong SG, Levin WJ, Ullrich A, McGuire WL. Human breast cancer: correlation of relapse and survival with amplification of the HER-2/neu oncogene. Science. 1987; 235:177-82.

5. Perou CM, Sorlie T, Eisen MB, van de Rijn M, Jeffrey SS, Rees CA, Pollack JR, Ross DT, Johnsen H, Akslen LA, Fluge O, Pergamenschikov A, Williams C, et al. Molecular portraits of human breast tumours. Nature. 2000; 406:74752. https://doi.org/10.1038/35021093.

6. Hanahan D, Weinberg RA. Hallmarks of cancer: the next generation. Cell. 2011; 144:646-74. https://doi. org/10.1016/j.cell.2011.02.013.

7. Warburg O. The metabolism of carcinoma cells. J Cancer Res. 1925; 9:148-63.

8. DeBerardinis RJ, Mancuso A, Daikhin E, Nissim I, Yudkoff M, Wehrli S, Thompson CB. Beyond aerobic glycolysis: transformed cells can engage in glutamine metabolism that exceeds the requirement for protein and nucleotide synthesis. Proc Natl Acad Sci U S A. 2007; 104:19345-50. https://doi.org/10.1073/pnas.0709747104.

9. Gámez-Pozo A, Berges-Soria J, Arevalillo JM, Nanni P, López-Vacas R, Navarro H, Grossmann J, Castaneda CA, Main P, Díaz-Almirón M, Espinosa E, Ciruelos E, Fresno Vara JA. Combined label-free quantitative proteomics and microRNA expression analysis of breast cancer unravel molecular differences with clinical implications. Cancer Res. 2015; 75:2243-53.

10. Gámez-Pozo A, Trilla-Fuertes L, Berges-Soria J, Selevsek N, López-Vacas R, Díaz-Almirón M, Nanni P, Arevalillo JM, Navarro H, Grossmann J, Gayá Moreno F, Gómez Rioja R, Prado-Vázquez G, et al. Functional proteomics outlines the complexity of breast cancer molecular subtypes. Sci Rep. 2017; 7:10100. https://oi.org/10.1038/ s41598-017-10493-w.
11. Jones NP, Schulze A. Targeting cancer metabolism--aiming at a tumour's sweet-spot. Drug Discov Today. 2012; 17:232-41. https://doi.org/10.1016/j.drudis.2011.12.017.

12. Zhou G, Myers R, Li Y, Chen Y, Shen X, Fenyk-Melody J, Wu M, Ventre J, Doebber T, Fujii N, Musi N, Hirshman MF, Goodyear LJ, et al. Role of AMP-activated protein kinase in mechanism of metformin action. J Clin Invest. 2001; 108:1167-74. https://doi.org/10.1172/JCI13505.

13. Beck JT. Potential role for mammalian target of rapamycin inhibitors as first-line therapy in hormone receptor-positive advanced breast cancer. Onco Targets Ther. 2015; 8:362938. https://doi.org/10.2147/OTT.S88037.

14. Varma A, Palsson BO. Parametric sensitivity of stoichiometric flux balance models applied to wild-type Escherichia coli metabolism. Biotechnol Bioeng. 1995; 45:69-79. https://doi.org/10.1002/bit.260450110.

15. Pramanik J, Keasling JD. Stoichiometric model of Escherichia coli metabolism: incorporation of growth-rate dependent biomass composition and mechanistic energy requirements. Biotechnol Bioeng. 1997; 56:398-421. https:// doi.org/10.1002/(SICI)1097-0290(19971120)56:4<398::AIDBIT6>3.0.CO;2-J.

16. Edwards J. Functional genomics and the computational analysis of bacterial metabolism. Department of Bioengineering. San Diego: University of California. 1999.

17. Edwards JS, Ibarra RU, Palsson BO. In silico predictions of Escherichia coli metabolic capabilities are consistent with experimental data. Nat Biotechnol. 2001; 19:125-30.

18. Schilling C, Palsson B. The underlying pathway structure of biochemical reaction networks. Proc Natl Acad Sci USA). 1998; 4193-8.

19. Asgari Y, Zabihinpour Z, Salehzadeh-Yazdi A, Schreiber F, Masoudi-Nejad A. Alterations in cancer cell metabolism: the Warburg effect and metabolic adaptation. Genomics. 2015; 105:275-81.

20. Wortham M, Czerwinski M, He L, Parkinson A, Wan YJ. Expression of constitutive androstane receptor, hepatic nuclear factor 4 alpha, and P450 oxidoreductase genes determines interindividual variability in basal expression and activity of a broad scope of xenobiotic metabolism genes in the human liver. Drug Metab Dispos. 2007; 35:1700-10. https://doi.org/10.1124/dmd.107.016436.

21. Motamedian E, Taheri E, Bagheri F. Proliferation inhibition of cisplatin-resistant ovarian cancer cells using drugs screened by integrating a metabolic model and transcriptomic data. Cell Prolif. 2017; 50:e12370. https:// doi.org/10.1111/cpr.12370.

22. Buler M, Aatsinki SM, Izzi V, Uusimaa J, Hakkola J. SIRT5 is under the control of PGC-1 $\alpha$ and AMPK and is involved in regulation of mitochondrial energy metabolism. FASEB J. 2014; 28:3225-37. https://doi.org/10.1096/fj.13-245241.

23. Gomes P, Outeiro TF, Cavadas C. Emerging Role of Sirtuin 2 in the Regulation of Mammalian Metabolism. Trends 
Pharmacol Sci. 2015; 36:756-68. https://doi.org/10.1016/j. tips.2015.08.001.

24. Lin ZF, Xu HB, Wang JY, Lin Q, Ruan Z, Liu FB, Jin W, Huang HH, Chen X. SIRT5 desuccinylates and activates SOD1 to eliminate ROS. Biochem Biophys Res Commun. 2013; 441:191-5. https://doi.org/10.1016/j. bbrc.2013.10.033.

25. Cuyàs E, Corominas-Faja $\mathrm{B}$, Joven $\mathrm{J}$, Menendez JA. Cell cycle regulation by the nutrient-sensing mammalian target of rapamycin (mTOR) pathway. Methods Mol Biol. 2014; 1170:113-44. https://doi. org/10.1007/978-1-4939-0888-2_7.

26. Tang LH, Contractor T, Clausen R, Klimstra DS, Du YC, Allen PJ, Brennan MF, Levine AJ, Harris CR. Attenuation of the retinoblastoma pathway in pancreatic neuroendocrine tumors due to increased cdk4/cdk6. Clin Cancer Res. 2012; 18:4612-20. https://doi.org/10.1158/1078-0432. CCR-11-3264.

27. Knudsen ES, Witkiewicz AK. Defining the transcriptional and biological response to $\mathrm{CDK} 4 / 6$ inhibition in relation to ER+/HER2- breast cancer. Oncotarget. 2016; 7:69111-23. https://doi.org/10.18632/oncotarget.11588.

28. Gonzalez J, Harris T, Childs G, Prystowsky MB. Rapamycin blocks IL-2-driven $\mathrm{T}$ cell cycle progression while preserving $\mathrm{T}$ cell survival. Blood Cells Mol Dis. 2001; 27:572-85. https://doi.org/10.1006/bcmd.2001.0420.

29. Wang XC, Tian J, Tian LL, Wu HL, Meng AM, Ma TH, Xiao J, Xiao XL, Li CH. Role of Cks1 amplification and overexpression in breast cancer. Biochem Biophys Res Commun. 2009; 379:1107-13. https://doi.org/10.1016/j. bbrc.2009.01.028.

30. Cui Y, Huang Q, Auman JT, Knight B, Jin X, Blanchard KT, Chou J, Jayadev S, Paules RS. Genomic-derived markers for early detection of calcineurin inhibitor immunosuppressant-mediated nephrotoxicity. Toxicol Sci. 2011; 124:23-34. https://doi.org/10.1093/toxsci/kfr217.

31. Pannu V, Rida PC, Ogden A, Turaga RC, Donthamsetty S, Bowen NJ, Rudd K, Gupta MV, Reid MD, Cantuaria G, Walczak CE, Aneja R. HSET overexpression fuels tumor progression via centrosome clustering-independent mechanisms in breast cancer patients. Oncotarget. 2015; 6:6076-91. https://doi.org/10.18632/oncotarget.3475.

32. Davies TH, Ning YM, Sánchez ER. Differential control of glucocorticoid receptor hormone-binding function by tetratricopeptide repeat (TPR) proteins and the immunosuppressive ligand FK506. Biochemistry. 2005; 44:2030-8. https://doi.org/10.1021/bi048503v.

33. Vilasco M, Communal L, Mourra N, Courtin A, Forgez $\mathrm{P}$, Gompel A. Glucocorticoid receptor and breast cancer. Breast Cancer Res Treat. 2011; 130:1-10. https://doi. org/10.1007/s10549-011-1689-6.

34. He H, Sun Y. Ribosomal protein $\mathrm{S} 27 \mathrm{~L}$ is a direct $\mathrm{p} 53$ target that regulates apoptosis. Oncogene. 2007; 26:2707-16. https://doi.org/10.1038/sj.onc.1210073.
35. Rosenbluth JM, Mays DJ, Jiang A, Shyr Y, Pietenpol JA. Differential regulation of the $\mathrm{p} 73$ cistrome by mammalian target of rapamycin reveals transcriptional programs of mesenchymal differentiation and tumorigenesis. Proc Natl Acad Sci USA. 2011; 108:2076-81. https://doi.org/10.1073/ pnas. 1011936108.

36. Belkaid A, Duguay SR, Ouellette RJ, Surette ME. $17 \beta$-estradiol induces stearoyl-CoA desaturase-1 expression in estrogen receptor-positive breast cancer cells. BMC Cancer. 2015; 15:440. https://doi.org/10.1186/ s12885-015-1452-1.

37. Boulay A, Rudloff J, Ye J, Zumstein-Mecker S, O'Reilly T, Evans DB, Chen S, Lane HA. Dual inhibition of mTOR and estrogen receptor signaling in vitro induces cell death in models of breast cancer. Clin Cancer Res. 2005; 11:531928. https://doi.org/10.1158/1078-0432.CCR-04-2402.

38. O'Reilly T, McSheehy PM, Wartmann M, Lassota P, Brandt R, Lane HA. Evaluation of the mTOR inhibitor, everolimus, in combination with cytotoxic antitumor agents using human tumor models in vitro and in vivo. Anticancer Drugs. 2011; 22:58-78. https://doi.org/10.1097/ CAD.0b013e3283400a20.

39. Yee KW, Zeng Z, Konopleva M, Verstovsek S, Ravandi F, Ferrajoli A, Thomas D, Wierda W, Apostolidou E, Albitar M, O'Brien S, Andreeff M, Giles FJ. Phase I/ II study of the mammalian target of rapamycin inhibitor everolimus (RAD001) in patients with relapsed or refractory hematologic malignancies. Clin Cancer Res. 2006; 12:5165-73. https://doi.org/10.1158/1078-0432. CCR-06-0764.

40. Andresen BS, Christensen E, Corydon TJ, Bross P, Pilgaard B, Wanders RJ, Ruiter JP, Simonsen H, Winter V, Knudsen I, Schroeder LD, Gregersen N, Skovby F. Isolated 2-methylbutyrylglycinuria caused by short/branchedchain acyl-CoA dehydrogenase deficiency: identification of a new enzyme defect, resolution of its molecular basis, and evidence for distinct acyl-CoA dehydrogenases in isoleucine and valine metabolism. Am J Hum Genet. 2000; 67:1095-103. https://doi.org/10.1086/303105.

41. Fingar DC, Richardson CJ, Tee AR, Cheatham L, Tsou C, Blenis J. mTOR controls cell cycle progression through its cell growth effectors S6K1 and 4E-BP1/eukaryotic translation initiation factor 4E. Mol Cell Biol. 2004; 24:200-16.

42. Tengku Din TA, Seeni A, Khairi WN, Shamsuddin S, Jaafar H. Effects of rapamycin on cell apoptosis in MCF-7 human breast cancer cells. Asian Pac J Cancer Prev. 2014; 15:10659-63.

43. Resendis-Antonio O, Checa A, Encarnación S. Modeling core metabolism in cancer cells: Surveying the topology underling the Warburg effect. PLoS One. 2010; 5:e12383.

44. Vázquez A, Liu J, Zhou Y, Oltvai ZN. Catabolic efficiency of aerobic glycolysis: the Warburg effect revisited. BMC Syst Biol. 2010; 4:58. https://doi.org /10.1186/1752-0509-4-58. 
45. Dai J, Liu M, Ai Q, Lin L, Wu K, Deng X, Jing Y, Jia M, Wan J, Zhang L. Involvement of catalase in the protective benefits of metformin in mice with oxidative liver injury. Chem Biol Interact. 2014; 216:34-42. https://doi. org/10.1016/j.cbi.2014.03.013.

46. Kukidome D, Nishikawa T, Sonoda K, Imoto K, Fujisawa K, Yano M, Motoshima H, Taguchi T, Matsumura T, Araki E. Activation of AMP-activated protein kinase reduces hyperglycemia-induced mitochondrial reactive oxygen species production and promotes mitochondrial biogenesis in human umbilical vein endothelial cells. Diabetes. 2006; 55:120-7.

47. Volarevic V, Misirkic M, Vucicevic L, Paunovic V, Simovic Markovic B, Stojanovic M, Milovanovic M, Jakovljevic V, Micic D, Arsenijevic N, Trajkovic V, Lukic ML. Metformin aggravates immune-mediated liver injury in mice. Arch Toxicol. 2015; 89:437-50. https://doi.org/10.1007/ s00204-014-1263-1.

48. Vannini F, Kashfi K, Nath N. The dual role of iNOS in cancer. Redox Biol. 2015; 6:334-43. https://doi. org/10.1016/j.redox.2015.08.009.

49. El-Mir MY, Nogueira V, Fontaine E, Avéret N, Rigoulet M, Leverve X. Dimethylbiguanide inhibits cell respiration via an indirect effect targeted on the respiratory chain complex I. J Biol Chem. 2000; 275:223-8.

50. Chou TC. Theoretical basis, experimental design, and computerized simulation of synergism and antagonism in drug combination studies. Pharmacol Rev. 2006; 58:62181. https://doi.org/10.1124/pr.58.3.10.

51. Stekhoven DJ, Bühlmann P. MissForest-nonparametric missing value imputation for mixed-type data. Bioinformatics. 2012; 28:112-8. https://doi.org/10.1093/ bioinformatics/btr597.

52. Huang W, Sherman BT, Lempicki RA. Systematic and integrative analysis of large gene lists using DAVID bioinformatics resources. Nat Protoc. 2009; 4:44-57. https://doi.org/10.1038/nprot.2008.211.

53. Abreu G, Edwards D, Labouriau R. High-Dimensional Graphical Model Search with the gRapHD R Package. J Stat Softw. 2010; 37: 1-18.
54. R Core Team. R: A language and environment for statistical computing. Vienna, Austria: R Foundation for Statistical Computing. 2013.

55. Schellenberger J, Que R, Fleming RM, Thiele I, Orth JD, Feist AM, Zielinski DC, Bordbar A, Lewis NE, Rahmanian S, Kang J, Hyduke DR, Palsson BØ. Quantitative prediction of cellular metabolism with constraint-based models: the COBRA Toolbox v2.0. Nat Protoc. 2011; 6:1290-307.

56. Thiele I, Swainston N, Fleming RM, Hoppe A, Sahoo S, Aurich MK, Haraldsdottir H, Mo ML, Rolfsson O, Stobbe MD, Thorleifsson SG, Agren R, Bölling C, et al. A community-driven global reconstruction of human metabolism. Nat Biotechnol. 2013; 31:419-25. https://doi. org/10.1038/nbt.2488.

57. Colijn C, Brandes A, Zucker J, Lun D, Weiner B, Farhat M, Cheng T, Moody B, Murray M, Galagan J. Interpreting expression data with metabolic flux models: Predicting Mycobacterium tuberculosis mycolic acid production. PLoS Comput Biol. 2009; 5:e1000489.

58. Barker BE, Sadagopan N, Wang Y, Smallbone K, Myers CR, $\mathrm{Xi} \mathrm{H}$, Locasale JW, Gu Z. A robust and efficient method for estimating enzyme complex abundance and metabolic flux from expression data. Comput Biol Chem. 2015; 59:98112. https://doi.org/10.1016/j.compbiolchem.2015.08.002.

59. Varma A, Palsson BO. Stoichiometric flux balance models quantitatively predict growth and metabolic by-product secretion in wild-type Escherichia coli W3110. Appl Environ Microbiol. 1994; 60:3724-31.

60. Schellenberger J. Monte Carlo simulation in Systems Biology. Bioinformatics and Systems Biology. 2010: 162.

61. Davis AP, Grondin CJ, Johnson RJ, Sciaky D, King BL, McMorran R, Wiegers J, Wiegers TC, Mattingly CJ. The Comparative Toxicogenomics Database: update 2017. Nucleic Acids Res. 2017; 45:D972-D8. https://doi. org/10.1093/nar/gkw838. 